\title{
Isolation and Characterization of Vibrio parahaemolyticus Organisms from River Sources Around Barisal District, Bangladesh
}

\author{
Serajum Monir ${ }^{1}$, Fazle Rabbi ${ }^{1}$, Chowdhury R. Ahsan ${ }^{2}$ and Naiyyum Choudhury ${ }^{1}$ \\ ${ }^{1}$ Department of Mathematics and Natural Sciences, BRAC University, Mohakhali, Dhaka 1213, Bangladesh, ${ }^{2}$ Department of Microbiology, University \\ of Dhaka, Dhaka 1000, Bangladesh.
}

\begin{abstract}
Vibrio parahaemolyticus is a halophilic bacterium and lives in coastal water and causes gastroenteritis in humans especially where the rate of seafood consumption is high. Although it is a marine organism, very less is known about its pandemic spreading in the fresh water environment across Bangladesh. The present study was, therefore, undertaken to isolate and investigate the virulence potential, as well as phenotypic and genotypic traits of $V$. parahaemolyticus strains occurring in the fresh water environments around coastal southern parts of Bangladesh. A total of 70 suspected colonies of $V$. parahaemolyticus were isolated from water, sediment and roots of water hyacinth samples collected from four different locations of Bishkhali river located in Barisal district of Bangladesh. Of the 70 colonies, fourteen isolates were confirmed as $V$. parahaemolyticus. PCR detection for the presence of tox $R$, trh and $t d h$ genes among these seven isolates using primers specific for tox $R$, trh and $t d h$ revealed that all seven were positive for tox $R$ but negative for trh or $t d h$ genes. The results indicate that the isolates under this study do not contain virulence properties that correlate to the ability of causing infection and diseases.
\end{abstract}

Key words: Vibrio parahaemolyticus, toxR, trh, $t d h$, virulence genes.

\section{Introduction}

Vibrio parahaemolyticus, a well-known human pathogen, is the leading cause of gastroenteritis due to the consumption of seafood, primarily raw or improperly cooked shellfish worldwide ${ }^{1-2}$. These organisms were first recognized as a potential food-borne pathogen by Fujino and coworkers in 1951 in Japan and caused approximately half of all food poisoning cases in Taiwan, Japan and several Southeast Asian countries ${ }^{3-4}$. It is also reported to be an important agent of travelers' diarrhoea and has also occasionally been associated with extra intestinal infections, including wounds ${ }^{5}$. Although $V$. parahaemolyticus is a halophilic bacterium and mostly occurred in sea water environment, however, these organisms have also found to occur in fresh water environments, where the salinity of the water is almost zero and the temperature is relatively low ${ }^{6}$.

Pathogenic strains of $V$. parahaemolyticus generally produce a thermostable direct haemolysin (TDH) that is associated with the Kanagawa phenomenon (KP) ${ }^{7}$ and/or thermostable direct haemolysin-related haemolysin (TRH). Both TDH and TRH are encoded by $t d h$ and $t r h$ genes respectively ${ }^{8-9}$. Molecular epidemiological studies employing the DNA probes specific to the $t d h$ and trh genes revealed that not only strains carrying the $t d h$ gene but strains carrying a trh gene or both genes are strongly associated with gastroenteritis. Thus, both TDH and TRH are considered important virulence factors of V. parahaemolyticus ${ }^{10}$. One or both of these genes are detected in most clinical strains of
$V$. parahaemolyticus but are uncommon (about 1\%) in environmental and food isolates. Since 1996, an increased incidence of gastroenteritis in many parts of the world has been associated with $V$. parahaemolyticus serotype O3:K6 ${ }^{11-12}$. The association of this "new" O3:K6 serotype with large-scale foodborne disease outbreaks in Taiwan, Laos, Japan, Thailand, Korea and the United States between 1997 and 1998 suggest these organisms may have an unusual capacity to be transmitted by foods and/or to cause human infection and has pandemic potential ${ }^{13}$.

In Bangladesh, V. parahaemolyticus was identified as the etiologic agent in $3 \%$ of adult cases of dysentery ${ }^{15}$. Strains of different serogroups having genetic markers for the serogroup O3:K6 of $V$. parahaemolyticus were reported to have been isolated from hospitalized gastroenteritis patients in the Dhaka city of Bangladesh. Recent outbreaks of O3:K6 causing food poisoning in the sub-continent ${ }^{14}$, however, calls for scientific attention for environmental surveillance along the coastal regions as well as the fresh water environments of Bangladesh. Although it is a marine organism, very little is known about its pandemic spreading in the fresh water environment across Bangladesh. The present study was, therefore, undertaken to isolate and investigate the virulence potential, as well as phenotypic and genotypic traits of $V$. parahaemolyticus strains occurring in the fresh water environments around coastal southern parts of Bangladesh.

Corresponding Author: 


\section{Materials and Methods}

\section{Sampling site and sampling method}

This study on the isolation of $V$. parahaemolyticus was carried out for a period of six months (March to August, 2012) from the Bishkhali river located in the Barisal district. Samples were collected every three months from four different places of Bishkhali river and the salinity of the river water, which was found to be $0 \%$ in every time. Therefore, a total of 12 samples (water, sediment and water hyacinth) were collected each time from these sources.

Alkaline peptone water (APW) was used to facilitate the growth of the bacteria. For this, one ml of the sample (water, sediment or water hyacinth) was mixed with $9 \mathrm{ml}$ of APW and was incubated at $37^{\circ} \mathrm{C}$ for $18 \mathrm{~h}$. Ten fold serial dilution of the enriched samples were made and were subsequently streaked on to thiosulphate citrate bile salt sucrose (TCBS) agar plates. Following over night incubation at $37^{\circ} \mathrm{C}$, large, mucoid, raised, green colonies, were selected as suspected $V$. parahaemolyticus organisms.

\section{Reference strain}

V. parahaemolyticus strain A11 (new clone of serotype O3:K6, harboring $t d h$ obtained from a patient in Aomori Prefecture in Japan in 2000) was used as a reference strain to compare the biochemical characteristics and gene analysis.

\section{Identification of $V$. parahaemolyticus}

Suspected isolates of V. parahaemolyticus were inoculated into triple sugar iron medium and nutrient gar media supplemented each with $0,3,6,8$ and $10 \% \mathrm{NaCl}$. Test strains showing alkaline slant and acid butt reactions in the triple sugar iron medium and no growth in $0 \%$ but growth in 3 to $8 \% \mathrm{NaCl}$, were identified as $V$. parahaemolyticus. The test strains were further confirmed by the presence of toxR gene. The reference $V$. parahaemolyticus strain A11 was used as the positive control in all the tests.

\section{DNA template preparation}

A single colony was picked and grown at $37^{\circ} \mathrm{C}$ for overnight in alkaline peptone water broth containing $3 \% \mathrm{NaCl}$. One ml of cell suspension was transferred to a microfuge tube and was centrifuged at 10,000 rpm for $5 \mathrm{~min}$. After the supernatant was removed, the pellet was re-suspended in $1 \mathrm{ml}$ of TE buffer and heated at $100^{\circ} \mathrm{C}$ for $5 \mathrm{~min}$. After centrifugation at $10,000 \mathrm{rpm}$ for 5 min, the supernatant was transferred to a new tube and was used as the template. The supernatant containing the genomic DNA was kept at $-20^{\circ} \mathrm{C}$ before being assayed.

Detection of toxR, tdh and trh genes by Polymerase Chain reaction $(P C R)$

PCR detection of toxR gene in the purified genome DNA from bacterial isolateswas performed with primer 1 (5'-AGCCCGCTTTTCTTCAGACATC-3') and primer 2 (5'-AACGAGTCTTCTGCATGGT-3’), D3 primer (5'-CCACTACCACTCTCATATGC-3') and D5 primer
(5'-GGTACTAAATGGCTGACATC-3') for tdhgene and R2 primer (5'-GGCTCAAAAATGGTTAAGCG-3') and R6 primer (5'-CATTTCCGCTCTCATATGC-3') for trh (trh1, trh2) gene. The resultant amplicons were $399 \mathrm{bp}, 251 \mathrm{bp}$ and $250 \mathrm{bp}$ for $t o x R, t d h$ and trh genesrespectively. The PCR mixture (25 $\mu$ l) consisted of $10 \mathrm{mM}$ Tris- $\mathrm{HCl}$ (pH 8.3), $50 \mathrm{mM} \mathrm{KCl,} 1.5 \mathrm{mM} \mathrm{MgCl}_{2}, 0.01 \mathrm{mM}$ EDTA, $0.1 \mathrm{mM}$ dithiothreitol, 0.05\% Tween 20, 0.05\% Nonidet P40, 5\% glycerol, $0.2 \mathrm{mM}$ each of the four deoxynucleoside triphosphates (dNTP mixture; Takara, Ohtsu, Japan), and $0.5 \mathrm{U}$ of Taq polymerase (Takara Ex Taq; Takara, Japan). The ampliûcation conditions were set at one cycle of $94^{\circ} \mathrm{C}$ for $5 \mathrm{~min}$, followed by 35 cycles of ampliûcation consisting of denaturation at $94^{\circ} \mathrm{C}$ for $1 \mathrm{~min}$, annealing at $55^{\circ} \mathrm{C}$ for $1 \mathrm{~min}$, and extension at $72^{\circ} \mathrm{C}$ for $1 \mathrm{~min}$, followed by one cycle of $72^{\circ} \mathrm{C}$ for $7 \mathrm{~min}$. The PCR products were separated by electrophoresis in a $2 \%$ agarose gel, stained with ethidium bromide and recorded using a gel documentation system (Bio Rad, USA).

\section{Results and Discussion}

Identification of V. parahaemolyticus is usually achieved through a series of biochemical tests after their growth and isolation on a selective medium. Several specialized selective bacteriological culture media have been developed for isolating vibrios. CHROM agar is used as a selective medium for $V$. parahaemolyticus. $V$. cholerae and $V$. alginolyticus which ferment sucrose, produce yellow colonies on TCBS, while $V$. parahaemolyticus and $V$. mimicus being sucrose non-fermenter, grow as green colonies. It has been demonstrated that tox $R$ gene, which has regulatory function in $V$. parahaemolyticus, is used for the specific identification $V$. parahaemolyticus.

On the TCBS agar plates, the suspected $V$. parahaemolyticus were found to be large, green, raised and mucoid colonies of approximately 2-3 mm in diameter. These separated colonies were then transferred to CHROM agar plate for further confirmation of the isolates as $V$. parahaemolyticus, where the colonies were found to be purple or violet colored. A total of 70 suspected colonies of $V$. parahaemolyticus were selected for biochemical tests.

The suspected 70 isolates were then subcultured onto alkaline peptone agar containing $3 \% \mathrm{NaCl}$ and were subjected to extensive biochemical tests, like salt tolerance test and triple sugar iron test (TSI). Only fourteen isolates those showed growth on the $\mathrm{NaCl}$ concentration of $3 \%, 6 \%, 7 \%$ and $8 \%$ and could not grow in $0 \%$ and $10 \%$ were identified as $V$. parahaemolyticus ${ }^{16}$. The suspected fourteen isolates also showed the reactions similar to the positive control strain in the triple sugar iron tests and were re-confirmed as $V$. parahaemolyticus.

All the fourteen confirmed $V$. parahaemolyticus isolates showed positive results in PCR, using toxR specific primers and yielded DNA bands of 399 bp in agarose gel electrophoresis (Figure 1). However, all these isolates were found to be negative for the virulent $t d h$ and trh genes. 


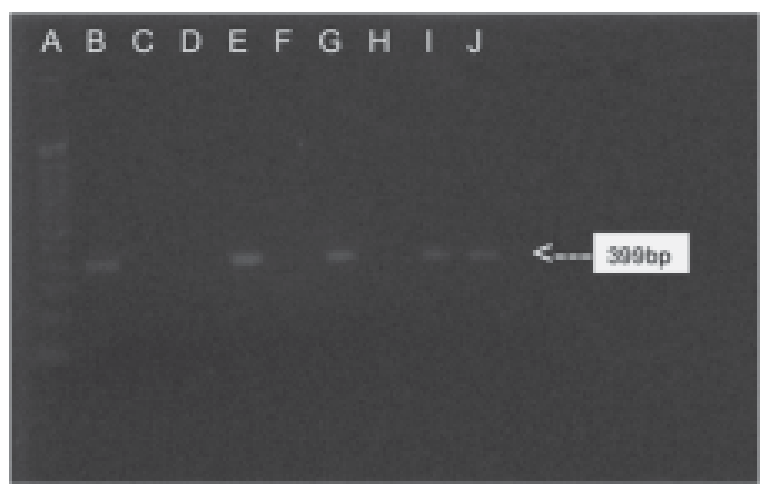

Figure 1. Agarose gel electrophoresis pattern of PCR amplicons of the seven confirmed $V$. parahaemolyticusisolates obtained with primers specific for toxR. Lane A, 100 bp DNA ladder; B to $H$, V. parahaemolyticus isolates; Lane I and J, positive control.

V. parahaemolyticus, a normal flora of the brackish and marine environment, has been considered as a common etiological agent of seafood-borne gastroenteritis and travelers' diarrhea in general $^{1-2}$ and to some extent as extra intestinal infections ${ }^{5}$. The thermostable direct haemolysin (TDH) or Kanagawa phenomenon associated haemolysin has been considered as the major virulence factor due to the exclusive detection of TDH (encoded by $t d h$ gene) among strains isolated from cases of gastroenteritis ${ }^{17-18}$. The toxR gene was found in $V$. parahaemolyticus and in other Vibrio species and its regulatory function was described ${ }^{24}$. The toxR gene therefore appears to be well conserved among the Vibrio species.

In this study, all the fourteen confirmed V. parahaemolyticus isolates showed positive results in PCR using primer specific to tox $R$ gene. However, none of these isolates gave positive results using primers specific to trh and $t d h$ gene. This is also consistent with the biochemical results of the seven isolates, as none of the test isolates was found to be urease positive, which is thought to be directly correlated with the production of thermostable direct haemolysin related haemolysin (TRH) encoded by trh gene ${ }^{19-20}$. This study only focuses strains isolated from Bishkhsli river of Barisal district, Bangladesh. Such an absence of virulent genes from the isolated strains of $V$. parahaemolyticus could therefore be correlated to their avirulent property. Previously this organism was isolated from water $^{6}$, plankton ${ }^{21}$, and fish ${ }^{6}$ of freshwater environments. Miyamoto et al., (1962) showed that V. parahaemolyticus could be isolated from seawater during the summer, but not during the winter ${ }^{22}$. Colwell (1973) reported that vibrios passed the winter in sediment and then are released from the bottom communities, attach to zooplanktons, proliferate as the temperature rises and the free $V$. parahaemolyticus in the water are released from plankton ${ }^{23}$. However, it remains to be unknown how the numerous cells of $V$. parahaemolyticus present in the summer disappear when the water temperature drops.
Further studies can be designed to understand the factors contributing to the survival of $V$. parahaemolyticus in rivers of $0 \%$ salinity and to compare the clonal variation between clinical and environmental isolates belonging to the same serogroup. $V$. parahaemolyticus infections have been assumed to be of environmental origin, but little work has been done to confirm this assumption which needs further investigation.

\section{References}

1. Barker W H. Jr, and Gangarosa E J. 1974. Food poisoning due to Vibrio parahaemolyticus. Annual. Rev. Microbial. 25: 75-81.

2. Tison D L., and Kelly M T. 1984. Vibrio species of medical importance. Diagn. Microbiol. Infect. Dis. 2: 263-276.

3. Joseph S W, Colwell R R, and Kaper J B. 1983. Vibrio parahaemolyticus and related halophilic vibrios. Crit. Rev. Microbiol. 10: 77-123.

4. Chiou A, Chen L-H, and Chen S-K. 1991. Foodborn illness in Taiwan, 1981-1989. Food Aust. 43: 70-71.

5. Blake P A, Weaver R E, and Hollis D G. 1980. Diseases of humans (other than cholera) caused by vibrios. Annu. Rev. Microbial. 34: 341-367.

6. Sarkar BL, Nair GB, Banerjee AK and Pal SC. 1985. Seasonal distribution of Vibrio parahaemolyticus in freshwater environs and in association with freshwater fishes in Calcutta. Appl. Environ. Microbiol. 49: 132-136.

7. Honda T, Ni Y X and Miwatani T. Infect. Immun. 1988, 56(4):961.

8. Honda T, and Iida T. 1993. The pathogenicity of Vibrio parahaemolyticus and the role of thermostable direct hemolysin and related hemolysins. Rev. Med. Microbiol. 4: 106-113.

9. Nishibuchi M, and Kaper JB. 1990. Duplication and variation of the thermostable direct hemolysin ( $t d h)$ gene in Vibrio parahaemolyticus. Mol. Microbiol. 4: 87-99.

10. Shirai H, Ito H, Hirayama T., Nakabayashi Y, Kumagai K, Takeda Y., and Nishibuchi M. 1990. Molecular epidemiologic evidence for association of thermostable direct hemolysin (TDH) and TDH-related hemolysin of Vibrio parahaemolyticus with gastroenteritis. Infect. Immun. 58: 3568-3573.

11. Chiou C-S, Hsu S-Y, Chiu S-I, W T-K, and Chao C-S. 2000. Vibrio parahaemolyticus serovar O3:K6 as cause of unusually high incidence of food-born disease outbreaks in Taiwan from 1996 to 1999. J. Clin. Microbial. 38(12): 4621-4625.

12. Okuda J, Ishibashi M, Hayakawa E, Nishino T, Takeda Y, Mukhopadhyay A K, Grag S, Bhattacharya SK, Nair GB, and Nishibuchi M. 1997. Emergence of a unique clone of Vibrio parahaemolyticus in Calcutta, India, and isolation of strains from the same clonal group from Southeast Asian travelers arriving in Japan. J. Clin. Microbiol. 35(12): 3150-3155.

13. Matsumoto C, Okuda J, Ishibashi M, Iwanaga M, Grag P, Ramamurthy T, Wong H-C, Depaola A, kim YB, Albert M J, and Nshibuchi M. 2000. Pandemic spread of an O3:K6 clone of Vibrio parahaemolyticus and emergence of related strains evidence by arbitrarily primed PCR and toxRS sequence analysis. J. Clin. Microbiol. 38: 578-585.

14. Wong H-C, Liu S-H, and Lee B-K. 2000.Characteristics of Vibrio parahaemolyticus O3:K6 from Asia Appl. Env. Microbiol. 66(9): 3981-3986.

15. Carpenter CC J. 1990. Other pathogenic vibrios. p. 1647. In Gerald L. Mandell R. Gordon Douglas, and John E. Bennet. Principles and Practice of infectious Diseases. $3^{\text {rd }}$ ed. Vol. 2. Churchill Livingstone, New York. 
16. Venkateswaran K, Nakano H, Okabe T, Takayama K, Matsuda O, and Hashimoto H. 1989. Occurrence and distribution of Vibrio spp., Listonella spp., and Clostridium botulinum in the Seto Inland Sea of Japan. Appl. Environ. Microbiol. 55: 559-567.

17. Miyamoto Y, Kato T, Obara Y, Akiyama S, Takizawa K, and Yamai S. 1969. In vitro hemolytic characteristic of Vibrio parahaemolyticus: its close correlation with human pathogenicity. J. Bacteriol. 100: 1147-1149.

18. Sakazaki R, Tamura K, Kato T, Obara Y, Yarnai S, and Hobo K. 1968. Studies on the enteropathogenic, facultatively halophilic bacteria, Vibrio parahaemolyticus. III. Enteropathogenicity. Jpn. J. Med. Sci. Biol. 21: 325-331.

19. Suthienkul O, Ishibashi M, Iida T, Nettip N, Supavej S, Eamppokalap B, Makino M, and Honda T. 1995. Urease production correlates with possession of the trh gene in Vibrio parahaemolyticus strains isolated in Thailand. J. Infect. Dis. 172: 1405-1408.
20. Iida T, Suthienkul O, Park K S, Tang G Q, Yamamoto R K, and Ishibashi M. 1997. Evidence for genetic linkage between the ure and trh genes in Vibrio parahaemolyticus. J. Med. Microbiol. 46: 639645 .

21. Sarkar B L, Nair G B, Sircar B K, and Pal S C. 1983. Incidence and level of Vibrio parahaemolyticus associated with freshwater plankton. Appl. Environ. Microbiol. 46: 288-290.

22. Miyamoto Y, Nakamura K, and Takizawa K. 1962. Seasonal distribution of Oceanomonas spp., halophilic bacteria, in the coastal sea. Its significance in epidemiology and marine industry. Jpn. J. Microbiol. 6: 141-158.

23. Colwell R R, Kaneko T, Staley T, Sochard M, Pickar J, and Wan L. 1974. Vibrio parahaemolyticus - taxonomy, ecology and pathogenicity. p. 169-176. In T. Fujino, G. Sakaguchi, R. Sakazaki and Y. Takeda (ed.), International Symposium on Vibrio parahaemolyticus. Saikon Publishing Co., Tokyo. 